\title{
Spectrum sensing performance analysis based on primary user probability access/leave model
}

\author{
Song Chen ${ }^{1}$,ShuainanGu${ }^{1}, \mathrm{Ke} \mathrm{Li}^{1}$, and Peng Dong ${ }^{1}$ \\ ${ }^{1}$ Zhengzhou Institute of Information Science and Technology, Zhengzhou 450001, China
}

\begin{abstract}
Keywords: cognitive radio; spectrum sensing; detection probability; probability accessing; probability leaving
\end{abstract}

\begin{abstract}
In cognitive radio system, secondary user's self-sensing properties to the spectrum occupy is the start and basis of the whole cognitive process. The classical analysis of self-sensing properties has the hypothesis that the Primary user signal occupies the whole sensing slot. However, in the actual, primary user may access or leave anytime in the sensing slot. This paper does some discussion and analysis at this fact. Firstly, model according to behavior characteristics of primary user's probability accessing and leaving. Secondly, derive the probability of primary user's different length of sample points' appearance in sensing slot. Meanwhile, derive the function expression of secondary user's average detection probability theoretically in the theoretical framework of energy detection. Finally, the results of numerical simulation verify the rationality of theoretical derivation. Compared with the classical method, the behavior of primary user's probability accessing and leaving not only changes the trend of self-sensing properties, but also makes detection probability of secondary user reduced by more than $50 \%$.
\end{abstract}

\section{Introduction}

With the rapid growth of mobile communication services, Contradiction between radio spectrum resources shortage and information transmission requirements is becoming more and more significant. While searching for new communication resources, how to utilize existing spectrum resource has become a research focus. Cognitive radio technology can improve the spectrum utilization efficiency without changing the existing spectrum allocation, which has drawn wide attention in the research field. In a large amount of literature, system performance of PU's preferred 'sensing-transmission' system was analyzed and optimized.

In [1], according to the probability accessing problem of PU and SU's MAC. Cross Layer relationship model was built, where the maximization problem of SU system's throughput was changed to a nonlinear constraint optimization problem. Finally, a cross layer optimization algorithm was proposed to obtain the maximum system capacity.In[2], model for PU channel occupancy state by two-state continuous-time Markov chain. Adjust the 'perception - transmission' data frame structure of SU based on convex optimization and finally obtain the optimal network throughput of SU.In[3], phase and modulation were introduced. A new method was put forward to set the decision threshold, which improve the probability of detection energy detection effectively.

In the literature above, optimization analysis of system capacity and spectrum efficiency was made for sensing method for cognitive system. However, when it relates to detection performance analysis, make the default assumption that signals of PU occupy the whole sensing slot of SU.In[4], an algorithm was proposed in which weights of sampling points were set according to the PU's appearance probability. SU's single detection probability was improved with lower complexity. However, not only PU is accessed probability but also its occupation time of spectrum may also be probability.

In view of the question above, this paper has carried on the research and analysis focusing on the introduction of PU probability "access / leave" after introducing the model of the SU spectrum sensing performance.First of all, modeling probability on the "access/left" behavior according that the PU's arrival interval and service duration obeys behavior of the independent exponential distribution;Secondly, launching the probability function of different access / exit scene through the use of the memoryless property of the exponential distribution ; Then we obtained PU through 
the theory inferential reasoning to be stochastic "access / leave" under the model the SU average detection probability function expression based on the energy examination method.Finally, the model proposed in this paper and inference conclusion were verified and analyzed by numerical simulation which was compared with theclassical energy detection methods on perceived performance.

\section{System model}

\section{PU Access/Leave Model}

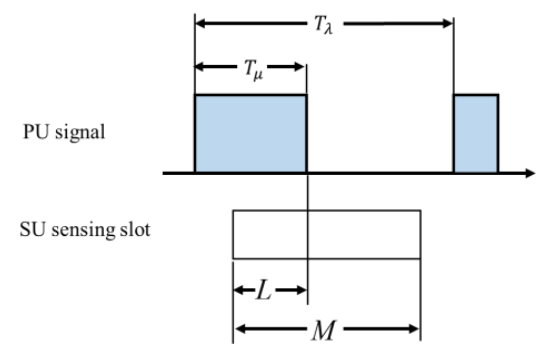

Fig. 1 PU access model

Assume that the time of arrival interval of PU and the length of time when PU occupies spectrum obey the exponential distribution with parameter $\lambda$ and $\mu$ respectively which are mutually independent[5]. Only one PU can access the spectrum each time, and if the current service of the system is available, the new PU can access directly, otherwise, the connection should be aborted.

We always suppose that the covered signal can occupy the entire detection slot of SU in the classical SU signal detection analysis. However, this papertakessuchsituation into account that PU can accesses or leavesprobability, which may occurjust as the scene shown in Fig. 1, i.e., the PU signal occupies only part samples of the sensing slot. Different situations will be analyzed in the later chapters of this article.

\section{PU Signal Model}

Considering the situation shown in Fig. 1, the PU signal received by SU does not fill the M samples in the whole sensing slot under the condition of $\mathrm{HB}$, only to appear $\mathrm{L}$ samples. Then the binary detection model of PU signal can be expressed as

$$
y_{i}= \begin{cases}s_{i}+n_{i} & H_{B}, i \in U \\ n_{i} & H_{I} \text { or } H_{B}, i \notin U\end{cases}
$$

Where, HB means that spectrum is occupied by PU in SU's sensing slot(Busy), while HI donates the spectrum is idle in the whole sensing slot of SU. $U$ is a set of samples in the length of $L$ where PU signal occurs.

We can assume that Si satisfies the complex Gaussian distribution with parameters of variance $\gamma$ and mean zero in this paper, and the samples ni of noise satisfies the same distribution with mean zero and variance 1 , then the variance ${ }^{\gamma}$ of Si can be considered as the signal to noise ratio (SNR) numerically [4].

\section{Analyses of Spectrum Sensing Performance}

\section{Relationship between PU Probability Access/Leave and Sensing Slot}

Assuming that the length of PU signal is $\mathrm{L}$ in the sensing slot with an overall length of $\mathrm{M}$, the probability of the occurrence of PU signal can be analyzed under 3 different situations according to the different places where PU signal occurs. Specific analysis and explanation of each scene is given in the following.

Scene 1

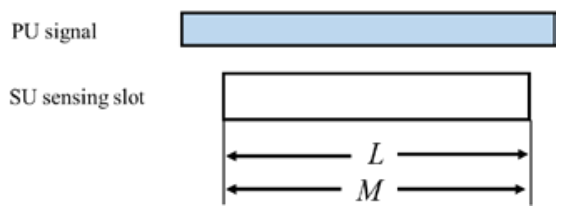


Fig. 2 PU probability access scene 1

Fig.2 shows the classic assumptive scene in which PU has occupied the spectrum the moment sensing slot begins, i.e. $\mathrm{P}(\mathrm{X}>0)$, and spectrum will be occupied by $\mathrm{PU}$ during the whole sensing slot. According to the lack-of-memory property of exponential distribution, the probability of PU's spectrum occupancy time length can be written as follows:

$$
P\left(T_{\mu}>M\right)=1-F_{\mu}(M)
$$

Where, $\mathrm{F}_{\mu}(L)$ means the CDF of time that the spectrum being occupied.Then the probability of the scene shown in Fig. 2 can be expressed by

$$
P_{1}(L)=P(X>0) \times P\left(T_{\mu}>M\right)(3)
$$

Scene 2

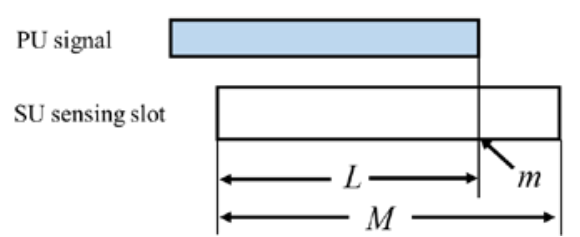

Fig. 3 PU probability access scene 2

Fig. 3 shows that PU has occupied the spectrum when sensing slot begins, that is $P(X>0)$. PU has been last $L$ samples during the sensing slot, and released the spectrum at the $m$ samples before the slot ended, which means $L=m$. Then the probability of sampling length being $L$ is

$$
P\left(T_{\mu}=L\right)=\left\{\begin{array}{lr}
F_{\mu}(1) & L=1 \\
F_{\mu}(L)-F_{\mu}(L-1) & 2 \leq L \leq M
\end{array}\right.
$$

Then the probability of the scene shown in Fig. 3 can be written as

$$
P_{2}(L)=P(X>0) P\left(T_{\mu}=L\right)(5)
$$

Scene 3

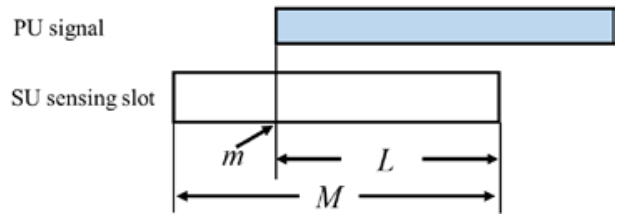

Fig. 4 PU probability access scene 3

Assume that the spectrum is in the idle state at the beginning of the sensing slot, i.e. $\mathrm{P}(\mathrm{X}=0)$. PU's occurrence at the $m$ th sample is equivalent to there being no PU access during the period of time $T_{\lambda}=(m-1) \tau$. Set $F_{\lambda}\left(T_{\lambda}\right)$ being theCumulative Distribution Function (CDF) of the idle time length of the authorized spectrum, and according to the lack-of-memory property of exponential distribution, the probability that PU signal occurs at the $m$ th sample in the sensing slot can be described as

$$
P_{\lambda}(m)=\left\{\begin{array}{lc}
F_{\lambda}(1) & m=1 \\
F_{\lambda}(m)-F_{\lambda}(m-1) & 2 \leq m<M
\end{array}\right.
$$

Since $m=M-L$,the probability of the scene shown in Fig. 3 is

$$
P_{3}(L)=P(X=0) \times P_{\lambda}(M-L) \times P\left(T_{\mu}>L\right)(7)
$$

Compositing the above three scenes, we can get the probability of the length of PU signal in the sensing slot being $L$

\section{Perceived performance analysis}

$$
P_{P U}(L)=\sum_{j=1}^{3} P_{j}(L)(8)
$$

According to the analysis of the previous section, the probability access/leave makes the length of the PU signal probability.In order to be able to reflect the PU "probability access/leave" impact on SU detection performance better. From the consideration on the statistical significance, the further analysis of the average detection probability of the $\mathrm{SU}^{\overline{P_{d}}}$ is as below: 


$$
\overline{P_{d}}=E\left[P_{d}(L)\right]=\frac{\sum_{L=1}^{M} P_{P U}(L) P_{d}(L)}{\sum_{L=1}^{M} P_{P U}(L)}=\frac{\sum_{L=1}^{M} \sum_{j=1}^{3} P_{j}(L) P_{d}(L)}{\sum_{L=1}^{M} \sum_{j=1}^{3} P_{j}(L)}
$$

Where $P_{d}(L)$ is the average detection probability when the sample length of PU is $L$. To facilitate the analysis and comparison,this paper adopted the energy detection method[6]to perceive PU signal.The energy detection model is as below when the sample length of PU is $L$ during the sense slot time.

Assuming that $n_{i}$ obey to zero mean and variance of 1 complex gaussianprobability distribution; $s_{i}$ obey to zero mean and variance of $\gamma$ complex gaussianprobability distribution. When the system is in status $H_{I}, Y_{L}$ obey to the center $\chi^{2}$ distribution, of which the degrees of freedomis $2 M$. When the system is in status $H_{B}, Y_{L}$ obey to the acentric $\chi^{2}$ distribution,of which the offset parameters is $\varphi=2 \sum_{i \in U}\left|s_{i}\right|^{2}$

$$
Y_{L} \square \begin{cases}\chi_{2 M}^{2}(\varphi) & H_{B} \\ \chi_{2 M}^{2} & H_{I}\end{cases}
$$

TheProbability Distribution Function of the offset parameters $\varphi$ [4]:

$$
f_{\varphi}(\varphi)=\frac{1}{2 \gamma L !}\left(\frac{\varphi}{2 \gamma}\right)^{L} e^{-\frac{\varphi}{2 \gamma}}, \quad \varphi \geq 0
$$

So the average detection probability of status $H_{B}$ is:

$$
P_{d}(L)=\int_{0}^{+\infty} Q_{M}(\sqrt{\varphi}, \sqrt{\eta}) f_{\varphi}(\varphi) d \varphi
$$

Where $Q_{M}(\square$,$) is Marcum Q-function[8]' \eta$ is the decision threshold of receiving signal. The paper [6] has given the derivation method and process of the closed function expression as formula(12), the closed expression of $P_{d}(L)$ can be derived through the simple variable substitution:

$$
\begin{aligned}
P_{d}(L)= & e^{-\frac{\eta V}{2}}\left(V^{L-1} L_{L-1}^{*}(-\omega)+(1-V) \sum_{i=0}^{L-2} V^{i} L_{i}^{*}(-\omega)\right) \\
& +V^{L} e^{-\frac{\eta}{2}} \sum_{i=1}^{M-1} \frac{1}{i !}\left(\frac{\eta}{2}\right)^{i}{ }_{1} F_{1}(L, i+1, \omega)
\end{aligned}
$$

Where $V=\frac{1}{1+\gamma}, \omega=\frac{\eta(1-V)}{2}, L_{i}^{*}(\square)$ is the Laguerre polynomial that obey to the order $i,{ }_{1} F_{1}(\square, \square)$ is confluent hypergeometric function. After the $P_{d}(L)$ has been known, the average detection probability can be derived through combining with formula(13) and formula(9):

$$
\begin{aligned}
\overline{P_{d}}= & \sum_{L=1}^{M} \sum_{j=1}^{3} P_{j}(L)\left[e^{-\frac{\eta V}{2}}\left(V^{L-1} L_{L-1}^{*}(-\omega)+(1-V) \sum_{i=0}^{L-2} V^{i} L_{i}^{*}(-\omega)\right)\right. \\
& \left.+V^{L} e^{-\frac{\eta}{2}} \sum_{i=1}^{M-1} \frac{1}{i !}\left(\frac{\eta}{2}\right)^{i}{ }_{1} F_{1}(L, i+1, \omega)\right] / \sum_{L=1}^{M} \sum_{j=1}^{3} P_{j}(L)
\end{aligned}
$$

\section{Simulation and analysis}

In this section, the derived results are compared and analyzed based on Matlab. The simulation conditions set as below: Setting theVoIPas the reference of the PU business strength[9], The probability of idle $P_{H_{I}}=0.65$.Assuming that false detection probability $P_{f}=0.1, \lambda=0.035, \mu=0.1$. In order to compare and analyze better, this paper take the classic energy detection method that the PU signal occupy the sense time slot as the comparative algorithm, of which the detection probability is marked with $P d_{M}$ in this figure, while the detection probability that used in this paper is marked with $P d_{L}$ : 


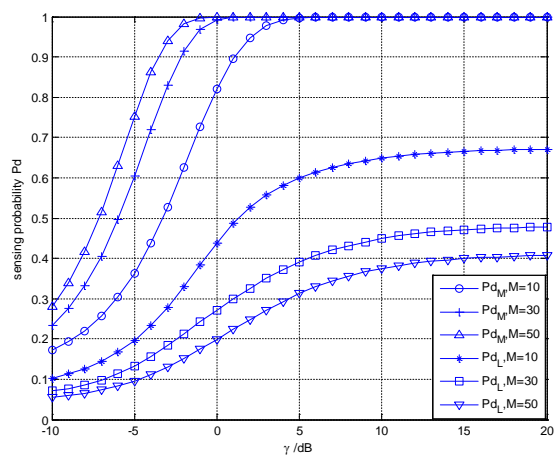

Fig.5 The comparison of the average detection probability with/without probability access in users

Fig. 5 shows that the relationship of the detection probability and the signal-to-noise ratio with/without the probability access of PU. When there is probability access of PU,the average detection probability is far lower than the classic method withoutprobability access. The average detection of the classic method increased with the increase of the length of the detection time slot $M$, while it's contrast in this paper. The phenomenon is because that the length of the PU signal is probability when there is probability access PU. The sample point without the PU signal is interference completely for detection process. When $M$ increase,the length of the disturbance also increase. So the average detection probability is reduced.

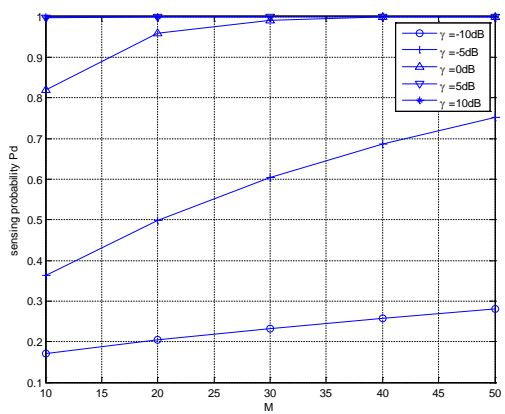

(a)

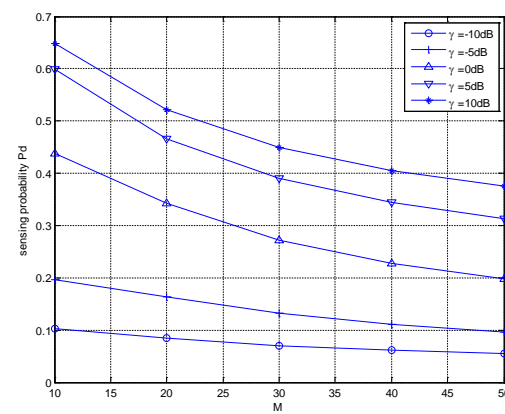

(b)

Fig.6The average detection probability in different length of time slot

Fig.6 (a) (b) shows the average detection probability in different length of time slot that derivedthrough the classic method and the method that used in this paper. We can infer thatthe average detection probability of the classic methodincreased with the increase of the length of the detection time slot $M$ under different signal-to-noise ratio, while it's contrast in this paper. The reason is because the unoccupied part is interference for judgment when the PU signal doesn't occupy the full sense time slot. When $M$ increase, the noise also increase, which produce more interference for judgment.

We can infer through the analysis of the results of numerical simulation that the detection performance of system has a huge change after the PU probability access/leave mechanism introduced in, compared with the classic method. At first,system detection performance decreased more than $50 \%$ because the effective PU signal sample points reduce. Then the noise produced by $L \leq M$ turned the relationship of the length of sense time slot and detection probability relations into the monotone decreasing. So when design and analysisthe system, the related parameters derived by the classic method would be false if there is probability access PU.

\section{Conclusion}

This paper is aimed at the condition that the PU signal doesn't occupy the sense time slot and the classic energy detection method can't be applied well. First of all, this paper build a single probability access/leave model of PU. And then the probability access/leave "can be divided into four scenarios for analysis, we can get the probability of the emergence of different scenarios by 
using the memoryless exponential distribution. At the same time, the detection probability can be derived based on related energy detection theory according to the length of the PU signal in the sense time slot. The last, we combine the probability of the emergence of four different scenarios and detection probability to deduce the closed expression of system average detection probability. And the derived results are verified by numerical simulation and analysis. But in this paper, we just research for the condition that single PU access/leave each time,and the research for multiple PU and SU will be the next step.

\section{Reference}

[1] Zheng S, Liang Y C, Kam P Y, et al. Cross-layered design of spectrum sensing and MAC for opportunistic spectrum access. 2009 Wireless Communications and Networking Conference. IEEE: Budapest, pp.1-6, 2009.

[2] Min A W, Kim K H, Singh J P, et al. Opportunistic spectrum access for mobile cognitive radios. 2011 IEEE Internationa Conference on Computer Communications. IEEE: Shanghai,pp. 2993-3001, 2011.

[3] Kale Sandikar R S, Wadhai V M, Helonde J B. Efficient spectrum sensing in cognitive Radio using energy detection method with new threshold formulation. Emerging Research Areas and 2013 International Conference on Microelectronics. IEEE:Kanjirapally, pp: 1-5, 2013.

[4] Ma J, Zhou X, Li G Y. Probability-based periodic spectrum sensing during secondary communication. IEEE Transactions on Communications, 58(4), pp. 1291-1301, 2010.

[5] Chu T, Phan H, Zepernick H. Dynamic Spectrum Access for Cognitive Radio Networks with Prioritized Traffics. IEEE Communications Letters, 18(7), pp.1218-1221, 2014.

[6] Digham F F, Alouini M S, Simon M K. On the energy detection of unknown signals over fading channels. IEEE Transactions on Communications, 55(1), pp.21-24, 2007.

[7] Xing Y, Chandramouli R, Mangold S, et al. Dynamic spectrum access in open spectrum wireless networks.IEEE Journal on Selected Areas in Communications, 24(3), pp. 626-637, 2006.

[8] Nuttall A H. Some integrals involving the Q-function. Naval Underwater Systems Center (NUSC) technical report, 1972.

[9] Hoven N, Tandra R, Sahai A. Some fundamental limits on cognitive radio. Wireless Foundations EECS, Univ. of California, Berkeley, 2005. 